\title{
Stellar dynamics of galactic nuclei with TIGER
}

\author{
Eric Emsellem \\ Sterrewacht Leiden, The Netherlands \\ Roland Bacon and Guy Monnet \\ Observatoire de Lyon, France
}

\begin{abstract}
We conducted an observational program using the TIGER integral field spectrograph to study the dynamical structure of nearby galactic nuclei. We already obtained new original results on three of the best "Black Holes Candidates": M 32, M 31 and M 104. Their nuclei exhibit complex morphologies and unusual dynamical properties such as: asymmetries, anisotropy, triaxiality which would have been impossible to detect with a "classical" spectrograph.
\end{abstract}

\section{Introduction}

The TIGER spectrograph was initially designed to study the dynamics of stellar systems, and particularly galactic nuclei. The aim of this program was to observe the central regions of "normal" (non active) galaxies to constrain the history of their formation and evolution, as well as to answer to some old and holy questions: do supermassive black holes exist in the center of galaxies? are ellipticals and bulges of spirals triaxial, anisotropic?

Stellar dynamics requires analysis of stellar absorption lines. This demands a high accuracy in the calibration step as well as a high overall transmission factor of the instrument (spatial elements being circles of only 0.39 arcsec in diameter). The results described in the following sections thus nicely illustrates the capability and power of such an instrument.

\section{Observations and Modelling}

Both photometry and spectroscopy (TIGER) of a sample of about ten nearby galactic nuclei were obtained since the November 1990 run. Multi band images (B, V, R, I) were taken with the use of the High Resolution Camera (HRCAM) available at the CFHT. The attained spatial resolution was typically 0.55 and 0.85 arcsec. FWHM for respectively the direct HRCAM images (squared pixels of $0.11 \operatorname{arcsec}^{2}$ ) and the 2D TIGER fields (microlenses of 0.39 arcsec. diameter).

A full description of the reduction steps is given in Bacon et al. (1994, hereafter B94). For the dynamics, we used a spectral domain around the magnesium triplet $(\sim 5160 \AA)$ including Fe 5270 and Fe 5335. Various techniques of spectral deconvolution to recover the full Line Of Sight Velocity Distribution 
(LOSVD) were applied (e.g. FCQ - Bender 1990, EXO - Rix \& White 1992), all giving very comparable values for $V$ (velocity), $\sigma$ (dispersion), and the higher order moments (Gauss-Hermite: $h_{3}, h_{4}$ ).

The results reported here concern three "black hole candidates", namely M 32, M 31 and M 104, respectively a compact elliptical, a large spiral, and a spiral-lenticular. We emphasize here only the uncovered structural properties which needed the bidimensional coverage and high spatial resolution provided by the TIGER integral field spectrograph.

We have built photometric and dynamical models of the three galaxies using the MGE method (see Emsellem et al. 1994a and 1994b). This allows us to (seeing) deconvolve and deproject the observed surface brightness analytically, to recover a "realistic" simple expression of the corresponding spatial luminosity distribution (axisymmetric or triaxial system). Self-gravitating models are then constructed with the help of the Jeans Equations, to test the isotropy, triaxiality and the presumed presence of a central supermassive black hole...

\section{A jump in the third dimension}

\section{Triaxiality?: M32}

Historically, Fig. 1 of Bacon et al. (1991) presents the first stellar velocity field ever ${ }^{1}$ obtained. Interesting enough, the photometric and dynamical minor axis are tilted with respect to each other by about ten degres. In other words, we have detected a small velocity gradient of about $15 \mathrm{~km} / \mathrm{s}$ along the photometric minor axis, which gives evidence for a slightly triaxial structure ${ }^{2}$ of the central region. It is striking to note that other published dynamics of the very same object do not show such a minor axis rotation: this is presumably due to the fact that long-slit spectrography of $M 32$ were taken at a position angle of 70 degres (vdMarel et al. 1994), which is indeed the orientation of the outer minor axis, but displaced by 5 degres with respect to the nucleus minor axis.

This result, implying a minimum triaxiality of 0.93 (axis ratio between the intermediate and long axis), has to be confirmed with the new set of TIGER frames we obtained during the November 1993 run, using a $2 k \times 2 k$ CCD. However, and regardless of its reality, such a structure requires $3 D$ spectrography to be detected.

\section{Stationarity?: M31}

Light et al. (1974) discovered an asymmetry in the surface brightness distribution of the nucleus of $M 31$, confirmed, almost 20 years later, by the pre-COSTAR HST frames (Lauer et al. 1993), and by the 0.35 arcsec. resolution ground-based HRCAM data obtained by Nieto (B94). The nucleus shows a double-peaked morphology, the brightest point (P1) being resolved and offcentered by about 0.4 arcsec. $(\sim 1.4 \mathrm{pc})$ from the center of the outer (bulge) isophotes. The TIGER data allowed us to reconstruct the $2 \mathrm{D}$ stellar velocity and velocity dispersion fields of the 5 central arcsec. (see B94) which first confirm the rapid rotation

\footnotetext{
${ }^{1}$ See discussion at the end of the present paper

${ }^{2}$ or an intrisic tilt
} 

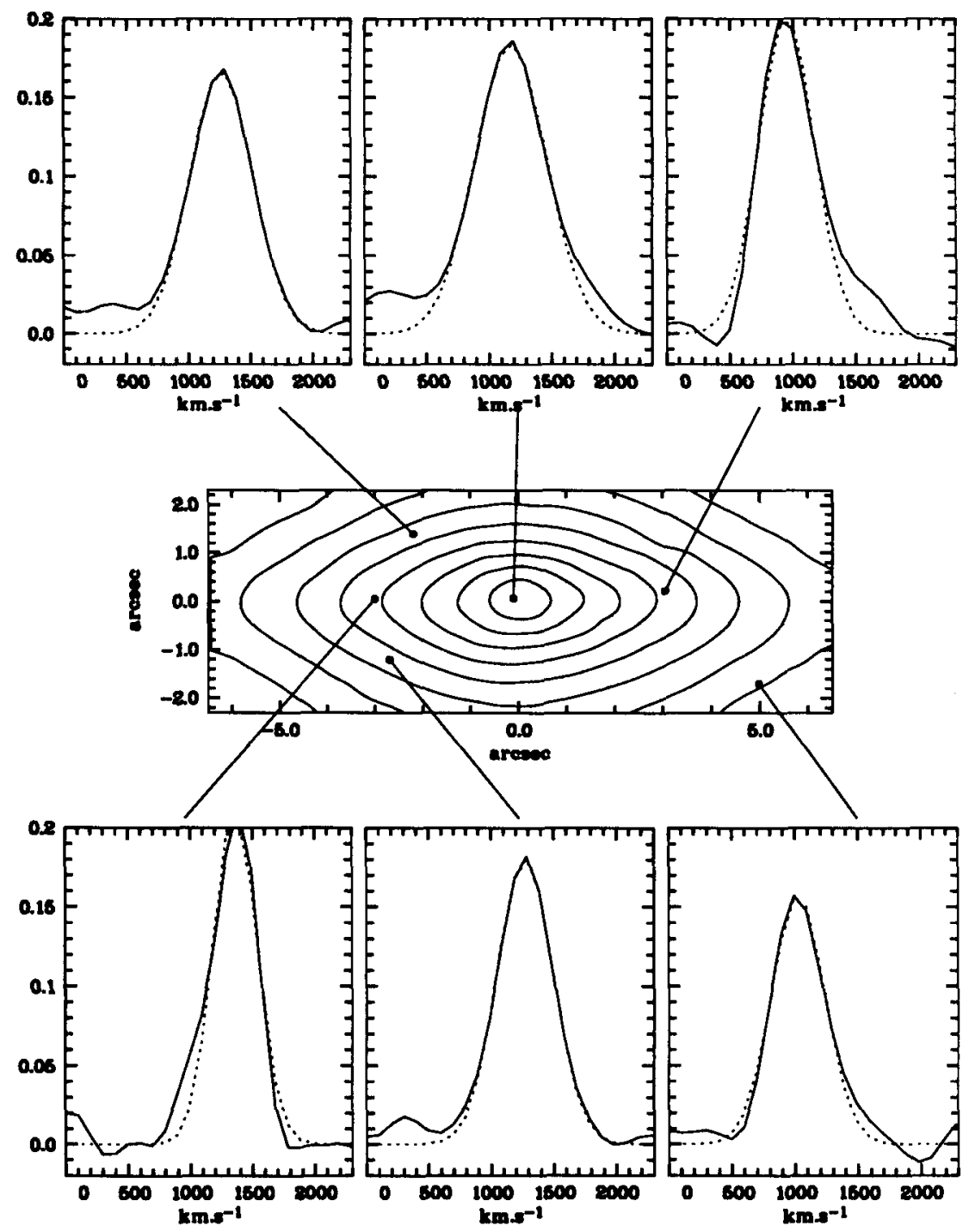

Figure 1. LOSVDs of M 104 at different points of the TIGER field, and the corresponding best gaussian fits (pointed lines): note the asymmetries of the profiles close to the major axis. The central panel shows the reconstructed TIGER image. 


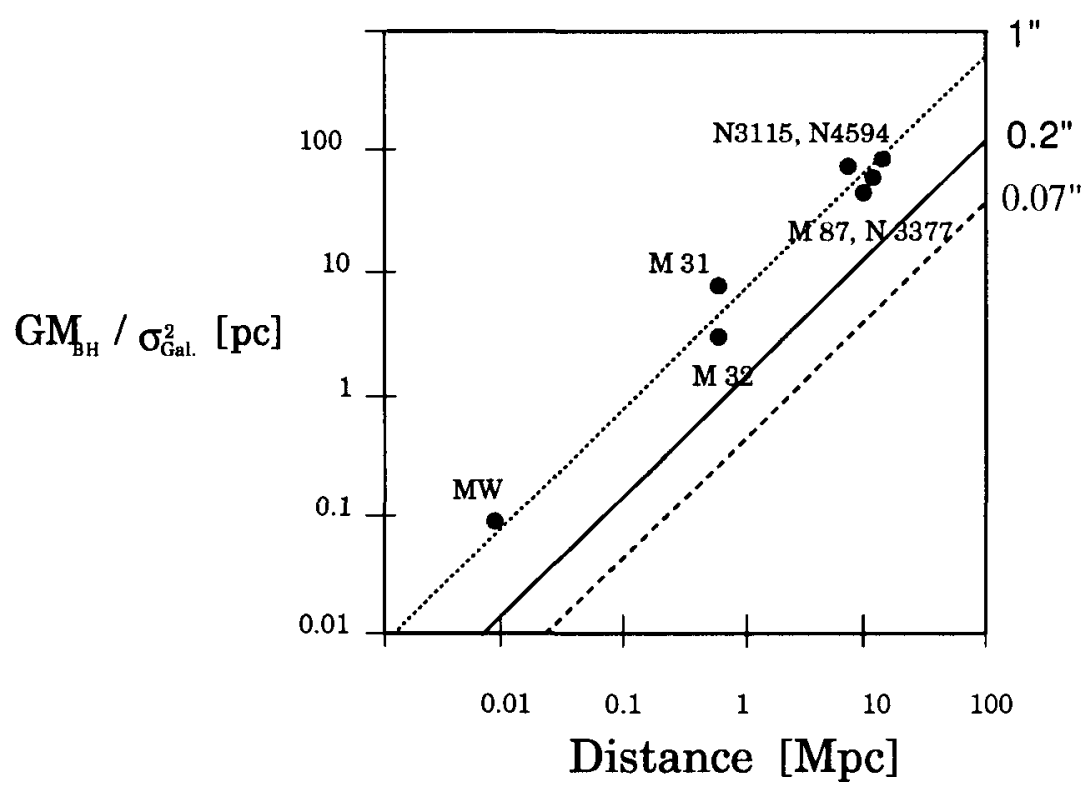

Figure 2. Black hole candidates drawn in a diagram "influence black hole radius vs. distance" showing the lines of 1 ", 0.2 " and 0.07 " resolution (after Rix).

$\left(260 \mathrm{~km} \cdot \mathrm{s}^{-1}\right.$ gradient) and the compactness (velocities basically going back to zero at 2-3 arcsec.) of the nucleus. The velocity field is almost symmetric, with a slightly higher velocity in the direction of the brighter point. A more surprising result resides in the dispersion field: the maximum lies at about 0.7 arcsec. $(2.4$ pc) but in the opposite direction with respect to P1. This has been found to be difficult to explain in terms of a second "cold" nucleus at P1, producing a dip in the dispersion field. Indeed, if the P1 corresponds to a swallowed secondary nucleus, we expect to observe a maximum of dispersion precisely at P1, and not on the opposite side (see B94 for further details). Jeans models are of little help in this case, since the distribution function is certainly non stationary. Thus, we conducted $\mathbf{N}$ body simulations (Combes et al., in prep.) which suggest that an offcentered black hole of $710^{7} \mathrm{M}_{\odot}$ does not move back to the very center of the nucleus through dynamical friction, and could drive a (only qualitatively) similar $m=1$ mode.

\section{... and LOSVD: $\mathrm{M} 104$}

The high resolution HRCAM images obtained on the Sombrero Galaxy confirmed the ring structure of the main disk, the presence of an inner disk, and revealed the presence of a bright nuclear disk. Self-gravitating dynamical models built from the corresponding MGE models (Emsellem et al. 1994b) showed, by comparison with previously published data (long-slit), that the disk is tangentially anisotropic (excess of streaming motions), and that the center presumably holds a central dark mass of about $1.010^{9} \mathrm{M}_{\odot}$. The four TIGER exposures 
covers a field of $12 \times 5$ arcsec. The velocity field exhibits a huge gradient of 500 $\mathrm{km} \cdot \mathrm{s}^{-1}$ and the dispersion field peaks at $270 \mathrm{~km} \cdot \mathrm{s}^{-1}$ in the center. The minor axis rotation observed by Kormendy (1988), and Peletier (1993) was probably due to a bad centering of their slit, since we do not detect any similar phenomenon (the center is measured a posteriori on the TIGER reconstructed image). The $2 D$ mapping of the LOSVDs clearly shows the presence of the inner disk whose contribution vanishes at about 5 arcsec.: the LOSVDs are quite asymmetric along the major axis where the inner disk dominates the photometry (Fig. 1). Again, Jeans Equation are of little use to understand the dynamics of M 104 nucleus: the derivation of its distribution function is needed.

\section{Distribution functions: towards the OASIS}

All these phenomena would have been difficult or even impossible to uncover with classical long-slit spectrography. At such spatial resolution, these nuclei reveals complex tridimensional structures which can hardly be understood with sole velocity and dispersion profiles.

Furthermore, it becomes clear now that we need to recover the complete distribution functions of these objects to understand their morphology and dynamics. For a two integral axisymmetric model, the surface brightness constrains only the even part of the distribution function. The derivation of the odd part requires the azimuthal streaming motion: in other terms a full bidimensional velocity field! $3 D$ spectrographs such as TIGER do provide the needed information.

As shown in Fig. 2, the case for the evidence of supermassive black holes in the center of galaxies is still very uncertain. The mounting of the new multi mode 3D spectrograph OASIS on the adaptive optics bonnette of the CFHT, with its 1500 spatial elements and 0.1 arcsec. resolution, will certainly be a decisive step towards the answer to such a debate.

\section{Discussion}

G. Cecil: A comment: beautiful work!

1- How do you account for spectral PSF variations through different lens-lets when you observe your velocity template stars? 2- How does the TIGER system influence the error-estimating procedures in your kinematic analysis (compared to those done in long-slit work)?

E. Emsellem: 1- We make several exposures of each template star, centered on different points of the lens-array. We then use either a merged spectrum or all obtained individual spectra depending on the importance of the measured PSF variations. 2-In fact, error-estimating procedures are quite independent of the instrument, as soon as the calibration step can be checked. We usually obtain an accuracy of a twentieth of a pixel using the regularly spaced peaks delivered by a fixed Fabry Perot etalon. Furthermore, the TIGER system has the great advantage to completely separate the spatial and the spectral stages, which is not the case in a long-slit spectrograph. 
J. Bland-Bawthorn: I would like to remind you that Wilkinson et al. (1986: MNRAS 218, 297) published an absorption line velocity map for NGC 5128. But, of course your data are by far the best to date. Your comment about nonstationary distribution functions is very interesting. This could arise through an off-centered black hole scattering the various orbit families.

E. Emsellem: You are absolutely right. Let's say M 32's velocity field was the first sub-arcsecond stellar velocity field ever obtained.

$P$. Stee: In your modelling you said that the dynamical friction is not powerful enough to move the black hole back to the very center. But you didn't include any gas in your simulation. So, do you think it can strongly affect this result?

E. Emsellem: We also obtained TIGER exposures of M31's nucleus in the spectral domain of [NII]-H $\alpha$ and did not detect any emission in the central 5 arcseconds. That means that the (ionized) gas in the nucleus (if any) has presumably no effect at all on the dynamics, except if the present structure of M31's nucleus has something to do with an evolutionary scheme, which would be anyway very difficult to take into account. Of course, for other galaxies (e.g. NGC 4594) the problem would be different...

\section{References}

Bacon, R., Emsellem, E., Nieto, J.-L., Monnet, G., 1991, CFH Bull. 26

Bacon, R., Emsellem, E., Monnet, G., Nieto, J.-L., 1994, A\&A, 281, 691

Bender, R., 1990, A\&A, 229, 441

Combes, F., Emsellem, E. , Bacon, R., 1994 in preparation

Emsellem, E., Monnet, G., Bacon, R., Nieto, J.-L., 1992, ESO/EIPC workshop, Elba

Emsellem, E., Monnet, G., Bacon, R., 1994a A\&A, in press

Emsellem, E., Monnet, G., Bacon, R., Nieto, J.-L., 1994b A\&A, in press

Kormendy, J., 1988 ApJ, 335, 40

Lauer, T. R., et al., 1993, AJ, 106, 1436

Light, E. S., Danielson, R. E., Schwarzschild, M., 1974, ApJ, 194, 257

Monnet, G., Bacon, R., Emsellem, E., 1992 A\&A, 253, 366

Peletier, R. F., 1993, A\&A, 271, 51

Rix, H.-W., White, S. D. M., 1992, MNRAS, 254, 389

van der Marel et al. 1994, sub. to MNRAS 